\begin{tabular}{|l|l|c|c|}
\hline Eiszeitalter u. Gegenwart & $\mathbf{2 8}$ & $\begin{array}{c}109-118 \\
8 \mathrm{Abb} ., 1 \mathrm{Tab} .\end{array}$ & Öbringen/Württ. 1978 \\
\hline
\end{tabular}

\title{
Glazigene Sichelmarken als Klimazeugen
}

\author{
(Nach Beobachtungen in Finnland) \\ Martin Schwarzbach*)
}

K u r z f a s s ung: In den Koli-Bergen (Nord-Karelien, Finnland) gibt es pleistozäne Rundhöcker aus massigen präcambrischen Quarziten. Die Rundhöcker sind ausgezeichnet aufgeschlossen und zeigen außer Gletscherschrammen auch - senkrecht dazu - zahlreiche bogenförmige glaziale Schürfmarken („friction cracks"). Solche bogenförmigen Marken sind zuerst aus Nordamerika ausführlich beschrieben und genetisch gedeutet worden, später aus Nordeuropa.

Am häufigsten sind in den Koli-Bergen Marken, bei denen die k o n k a ve Wölbung nach vorn („talab“, zur Gletscherzunge hin) zeigt. Sie sind z. T. als dünne, kaum eingetiefte Linien entwickelt („crescent fractures“, „Parabelrisse“); z. T. aber ist längs der Bogenlinie Gesteinsmaterial herausgebrochen, so daß ein mondsichel-ähnlicher Grundriß entsteht („lunate fracture“). Es wird vorgeschlagen, diese beiden Gruppen als konkave Risse bzw. konkave Sicheln zu bezeichnen. Es gibt Ubergänge zwischen beiden Typen. Die konkaven Risse treten häufig scharenweise und dicht gedrängt auf.

Seltener sind Sicheln, bei denen die kon vexe Seite nach vorn gerichtet ist („crescentic gouges", "Sichelbrüche"). Sie seien als konvexe Sicheln bezeichnet. - Alle bogenförmigen Marken kann man als Sichelmarken im weiteren Sinne (s. 1.) zusammenfassen, die konkaven und konvexen Sicheln als Sichelmarken im engeren Sinne (s. str.) (Tab. 1). Das zahlenmäßige Verhältnis von konkaven zu konvexen Marken ist in den Koli-Bergen gelegentlich von der Größenordnung 100:1.

Die eigentlichen Sicheln sind durch z w e i kreisförmige oder parabolische Bögen begrenzt. Der hintere Bogen ist die Schnittlinie zwischen der Oberfläche des Rundhöckers und einer Bruchfläche, die nach vorn flach geneigt ist (primärer Bruch, "principal fracture“). Der vordere, sekundäre Bogen wird durch einen steilen Bruch gebildet; dieser kann talauf oder talab gerichtet sein (Abb. 4). Die Sicheln s. str. zeigen daher zwar nicht durch die Orientierung des Bogens, wohl aber normalerweise durch die Neigung der primären Bruchfläche die Richtung der Eisbewegung an. Die konkaven Risse sind ebenfalls zur Rekonstruktion der Eisbewegung brauchbar, weil sie - im Gegensatz zu den Sicheln - eng gedrängt auftreten. Die sichelförmigen Marken sind also als Klimazeugen wertvoll (besonders für die präkänozoischen Vereisungen). Außerdem sind sie für das Problem des glazialen Geschiebetransports und der glazialen Exaration von allgemeinem Interesse.

\section{[Glacial crescentic marks as climatic indicators. According to observations in Finland]}

A bstract: In the Koli Mts. (northern Karelia, Finland) Pleistocene glacial whalebackforms show - besides of glacial striation - many exellently exposed crescentic friction cracks on the quartzitic rocks. Similar features were already described from North America and northern Europe. Both in the American and in the German literature, the conventional nomenclature for these marks is not very logical. In this paper, it is proposed 1. to differentiate between marks which are either concave or convex downstream; 2. between "fractures" ("Risse" in German) consisting of just a fracture, and "crescents" ("Sicheln" = sickels) due to removal of rock material, which consist of two fractures (a flat "principal fracture" and a steep one).

Old classification

(FLinT and others)

crescentic gouge

lunate fracture

crescentic fracture

\author{
New classification \\ (this paper) \\ convex crescent \\ concave crescent \\ concave fracture
}

Table 1 and fig. 4 schematically show the classification of the 3 most important marks. Most of the concave marks are concave fractures. In the Koli Mts., the rate between concave marks (mostly concave fractures) and convex crescents occasionally may reach about 100:1.

*) Anschrift des Verfassers: Prof. Dr. M. S c h w a r z b a c h, Geolog. Institut d. Universität, Zülpicher Str. 49, D-5000 Köln 1. 
The orientation of the bends alone does not unequivocally indicate the direction of ice-movement, but the flat inclination of the "principal fracture" in the crescents generally will do so. The concave fractures are mostly to be found in clusters; therefore clustering is another good criterion to allot these marks to concave ones and to determine in this way the direction of the glaciertongue.

Außer den häufigen und vielerwähnten, geradlinigen Gletscherschrammen, parallel zur Bewegungsrichtung des Eises, gibt es - viel seltener - auch bogen- oder sichelförmige glazigene Schürfmarken. Sie verlaufen que r zur Gletscherbewegung. Die Gletscherschrammen sind in bezug auf die Herkunftsrichtung des Eises meist zweideutig. Bei den Sichelmarken dagegen schien es eher möglich, in dieser Frage eine eindeutige Antwort zu erhalten. Aber die fast 100 Jahre alte Diskussion darüber gab keine sichere Entscheidung, und R. F. Flint kam zu der Feststellung (zuletzt 1971: 97): „In summary, neither the direction of concavity of a mark nor the dip of its fracture seems to be reliable evidence of direction of movement."

Mit den Sichelmarken im weitesten Sinne haben sich zuerst amerikanische Forscher eingehend beschäftigt, so CHAMBERLiN (1888), später vor allem GILBERT (1905), LAHEE (1912), Harris (1943) und in Canada Dreimanis (1953). Von Harris stammt auch die zusammenfassende Bezeichnung „friction cracks“ für alle bogenförmigen Risse oder Brüche im Gestein, die durch die Reibung zwischen Gletschereis und Gesteinsuntergrund (oder Geschieben) verursacht werden. Uber schwedische Vorkommen und ihre Genese berichtete ausführlich LJUNGNER (1930). Einzelbeobachtungen sind auch sonst aus Nordamerika und Nordeuropa veröffentlicht worden. Schulz $(1962,1967)$ kommt das Verdienst zu, für entsprechende Beispiele aus Mitteleuropa eine wertvolle Übersicht gegeben zu haben. Er mußte sich jedoch notgedrungen fast ganz auf Sichelmarken an erratischen Geschieben beschränken - fester Gesteinsuntergrund ist im norddeutschen Vereisungsgebiet eine Seltenheit, und außerdeutsche Vorkommen sind nicht jedem ohne weiteres zugänglich. Die Frage, ob man aus Sichelmarken die Herkunftsrichtung des Eises ableiten kann, läßt sich jedenfalls nur durch Beobachtungen am autochthonen Untergrund entscheiden.

Mir fielen bei einer Finnlandreise 1975 in den Koli-Bergen prachtvolle quarzitische Rundhöcker auf, die zahlreiche jungpleistozäne Sichelmarken zeigten. 1977 war ich für einige Tage wiederum in diesem Gebiet. Auf die Beobachtungen dort gründen sich die folgenden Ausführungen. Bereits Окко hatte 1950 einzelne finnische Beispiele beschrieben, aber auf die Koli-Berge nicht Bezug genommen.

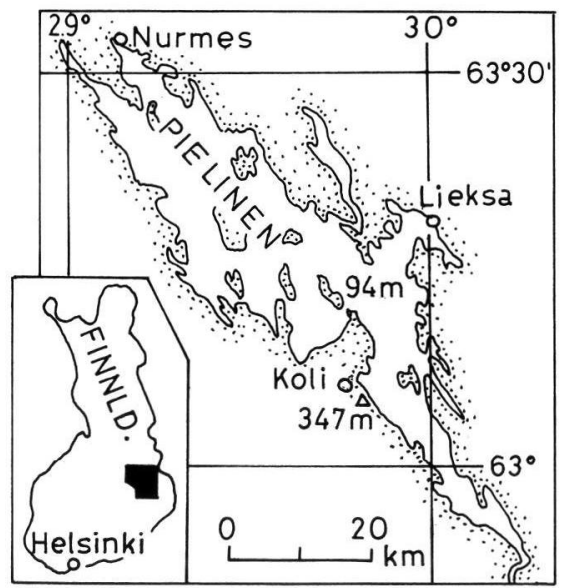

Abb. 1: Die Lage der Koli-Berge. - Situation-map of the Koli Mts. 


\section{Rundhöcker der Koli-Berge}

Die Koli-Berge liegen in Nord-Karelien unter $63^{\circ} 6^{\prime}$ nördl. Br., $29^{\prime} 48^{\prime}$ östl. Länge (Abb.1). Sie sind mit $347 \mathrm{~m}$ die höchsten Berge des südlichen Finnland und bilden eine Reihe von eis-überschliffenen Quarzitbuckeln, die gleichzeitig das ziemlich steile SW-Ufer des großen Pielinen-Sees bedingen. Der See-Spiegel liegt $94 \mathrm{~m}$ hoch.

Die weißen, massigen, oft nur undeutlich schichtigen Quarzite gehören zum präcambrischen Grundgebirge Finnlands. Gelegentlich zeigen Rippelmarken ihre sedimentäre Herkunft an.

Mindestens zum Teil fallen die Schichtflächen unter mittleren Winkeln nach SW hin ein. Ihrer nordöstlichen Streichrichtung entspricht der Verlauf des südwestlichen See-Ufers, und ebenso sind die langgestreckten Rundhöcker (und die Gletscherschrammen) ungefähr in dieser Richtung angeordnet. Manchmal liegen mehrere Rundhöcker parallel nebeneinander. Ihre äußere Formung ist offenbar weitgehend tektonisch bestimmt, d. h. durch die Streichrichtung der Quarzitschichten und auch durch steile Klüfte senkrecht dazu. Die Streichrichtung beeinflußte vielleicht auch die lokale Bewegungsrichtung des Eises, die allerdings auch generell in diesem Gebiet nach Untersuchungen der finnischen QuartärForscher NW-SE verläuft. Meist ist ein asymmetrisches Längsprofil der Rundhöcker deutlich, entsprechend der Eisbewegung; die Scheitellinie steigt in der Regel von NW nach SE hin an und bricht auf der Lee-Seite im SE (unter deutlicher Mitwirkung der Querklüfte) steil ab (Abb. 2). Doch gibt es auch Ausnahmen. Dazu gehört ein an $15 \mathrm{~m}$ langer Rundhöcker unmittelbar vor dem Hotel Ukko-Koli mit entgegengesetztem Längsprofil - ein isolierter Rundhöcker dieser Art könnte leicht zur Verwechslung von Stoß- und Lee-Seite und zur falschen Rekonstruktion der Eisbewegung führen! (Abb. 3).
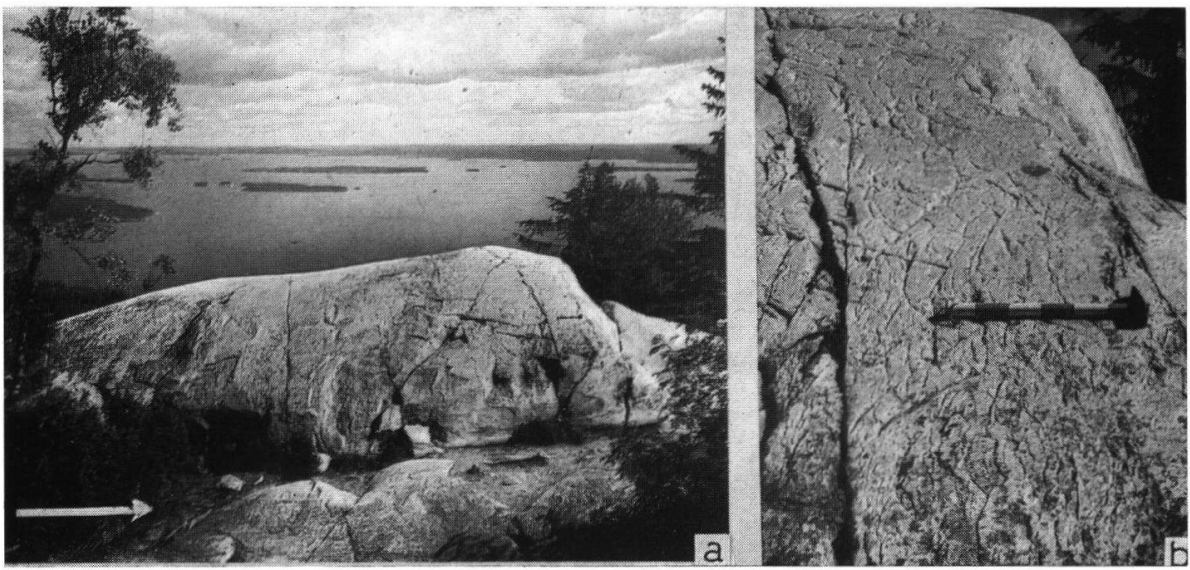

Abb. 2 a: Normal-asymmetrischer Quarzit-Rundhöcker der Koli-Berge. Im Hintergrund der See Pielinen. Die Flanke des Rundhöckers, die dem Beschauer zugewendet ist, entspricht ungefähr einer Schichtfläche (Einfallen ca. 40-50 SSW); einige Querklüfte senkrecht dazu. Kammlinie und (rechts) Hammer liegen parallel zur Eisbewegung (nach SE; von links nach rechts; weißer Pfeil). Zahlreiche Konkav-Risse (vgl. Abb. 2 b). - Quartzitic whaleback form with normal asymmetry. Lake Pielinen in the background. The flank facing the observer, approximately corresponds to a bedding plane of the quartzites (inclination c. 40-50 SSW); some joints to be seen perpendicular to the stratification. Sky-line and hammer (on the right side) parallel to ice-movement (towards $S E$, from left to right; white arrow). Many concave fractures (compare with fig. $2 b$ ).

Abb. 2 b: Detail vom Lee-Ende des Rundhöckers mit Konkav-Rissen. - Detail from the lee-end of the whaleback form with concave fractures. 


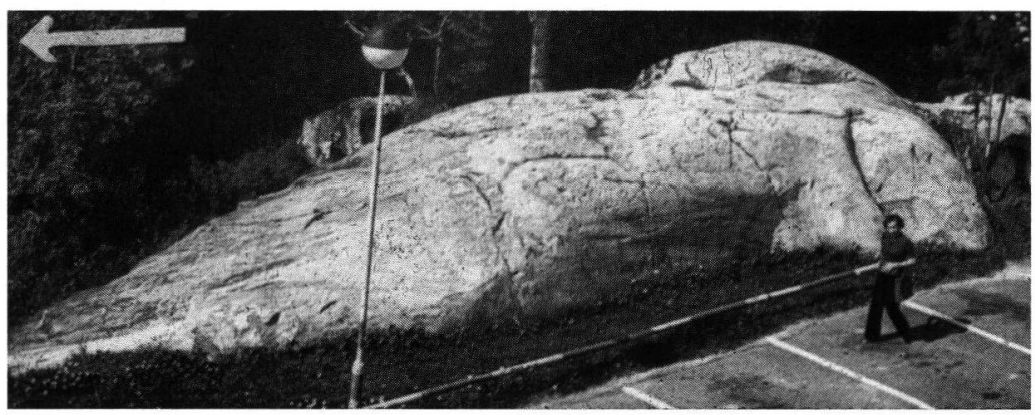

Abb. 3: Quarzit-Rundhöcker am Hotel Ukko-Koli. Anomale, irreführende Asymmetrie: StoßSeite (rechts) steil, Lee-Seite flach; das Eis kam von rechts (Pfeil). Der Rundhöcker trägt zahlreiche, verschiedenartige Sichelmarken. - Whaleback form from near the Hotel Ukko-Koli. Anomalous, misleading asymmetry: stoss-side (right) steep, lee-side flat; ice came from the right (arrow). Many, different crescentic marks exposed on the quartzitic rock.

Die Quarzitbuckel der Koli-Berge sind z. T. mit Flechten (und z. T. auch mit höheren Pflanzen) bewachsen, aber in der Gipfelregion z. T. völlig kahl und dort für das Studium der Schrammen und Schürfmarken am besten geeignet. Die „Spurensicherung“ - um einen aus der Kriminologie nur allzu gut bekannten Begriff zu gebrauchen — ist dort optimal. Die Häufigkeit von bogenförmigen Quermarken ist sehr verschieden. Es gibt Buckel ohne Marken, andere zeigen bis zu mehr als 100. Die schon von anderen hervorgehobene Häufung auf dem Kamm wird begünstigt dadurch, daß die Kammlinie in der Bewegungsrichtung des Eises in der Regel ansteigt. Auf den Längsseiten der Rundhöcker sind sichelartige Marken seltener, auf dem distalen Steilabfall fehlen sie. Es besteht also auch hier ein $\mathrm{Zu}-$ sammenhang zwischen größerer Häufigkeit der Marken und d e n Stellen, die dem stärksten Eisdruck ausgesetzt waren.

\section{Nomenklatur und Erscheinungsformen der Sichelmarken}

Die bogenförmigen oder ähnlich gestalteten Marken sind z. T. gut charakterisiert, z. T. aber unscharf gegeneinander abgegrenzt . Ihre systematische Typisierung ist daher schwierig, die Nomenklatur verwirrend, und LJUNGner hat aus diesem Grunde im Hinblick auf die sonst so verdienstvollen Untersuchungen CHAMBERLIN's und seine Einteilung mit Recht von einer "abschreckenden, scheinbaren Mannigfaltigkeit" gesprochen. Die Mannigfaltigkeit der Formen ist verständlich; denn bei der Entstehung der Marken wirken ja außer wechselnden primären Faktoren (wie vor allem dem Eisdruck und wohl auch der Einwirkung von großen Geschieben, die am Gletscherboden transportiert werden) auch sekundäre Einflüsse mit. Auch diese können von Ort zu Ort verschieden sein (z. B. die speziellen petrographischen Eigenschaften des Gesteinsuntergrundes, dessen Relief und Zerklüftung). Es empfiehlt sich also, nicht a 11 e möglichen Erscheinungsformen der Marken in ein Schema einzugliedern, sondern sich auf die zu beschränken, die klar definiert werden können. Das sind vor allem die wirklich bogenförmigen Marken. Untergeordnete Typen, wie z. B. die "chattermarks“ (von CHAMBERLIN mit den Vibriermarken einer Werkzeugmaschine verglichen) beiben also unberücksichtigt.

Als wichtigste Unterscheidungsmerkmale kann man heranziehen:

a) Die Orientierung der Bögen. Ihre konvexe Seite kann in der Bewegungsrichtung des Eises liegen, also nach vorn („talab“, zur Gletscherzunge hin) weisen. Aber 
die Bögen können auch umgekehrt orientiert sein, d.h. die konkave Seite nach vorn zeigen. In beiden Fällen sind die Bögen meist senkrecht zu den Gletscherschrammen orientiert.

b) Die Eintief ung der Marken. Viele Marken sind oberflächlich nur ganz wenig eingetieft (oft $<1 \mathrm{~mm}$ ). Sie erscheinen als dünne, linienartige Bögen. Man kann sie als $\mathrm{R}$ is $\mathrm{s}$ e bezeichnen.

Dagegen ist bei einer zweiten Gruppe Gesteinsmaterial deutlich sichtbar herausgebrochen. In diesen Fällen besteht jede Marke aus $z$ w e i ungefähr halbkreisförmigen Bögen, die sich wie bei einer schmalen Mondsichel aneinanderlegen. Der eine Bogen ist verursacht durch eine meist flach (ca. $20^{\circ}$ ) einfallende primäre Bruchfläche („principal fracture“,

Tab. 1:

Merkmale von Sichelmarken s. 1. - Characteristics of crescentic marks s. l.

\begin{tabular}{|c|c|c|c|}
\hline $\begin{array}{l}\text { Vorgeschlagene Nomenklatur } \\
\text { Proposed nomenclature }\end{array}$ & $\begin{array}{c}\text { Bisherige } \\
\text { amerikanische } \\
\text { Nomenklatur } \\
\text { Conventional } \\
\text { American } \\
\text { nomenclature }\end{array}$ & $\begin{array}{c}\text { Nach vorn } \\
\text { (talab): } \\
\text { Downstream: }\end{array}$ & $\begin{array}{l}\text { Gesteinsmaterial } \\
\text { herausgebrochen: } \\
\text { Removal of rock: }\end{array}$ \\
\hline konvexe Sichel convex crescent & crescentic gouge & $\begin{array}{l}\text { konvex } \\
\text { convex }\end{array}$ & \multirow{2}{*}{$\begin{array}{l}\text { deutlich } \\
\text { (Sichelmarken } \\
\text { s. str) } \\
\text { clearly } \\
\text { (crescentic marks } \\
\text { s. str) }\end{array}$} \\
\hline konkave Sichel concave crescent & lunate fracture & \multirow{2}{*}{$\begin{array}{l}\text { konkav } \\
\text { concave }\end{array}$} & \\
\hline concave fracture & crescentic fracture & & $\begin{array}{l}\text { kaum } \\
\text { scarcely }\end{array}$ \\
\hline
\end{tabular}

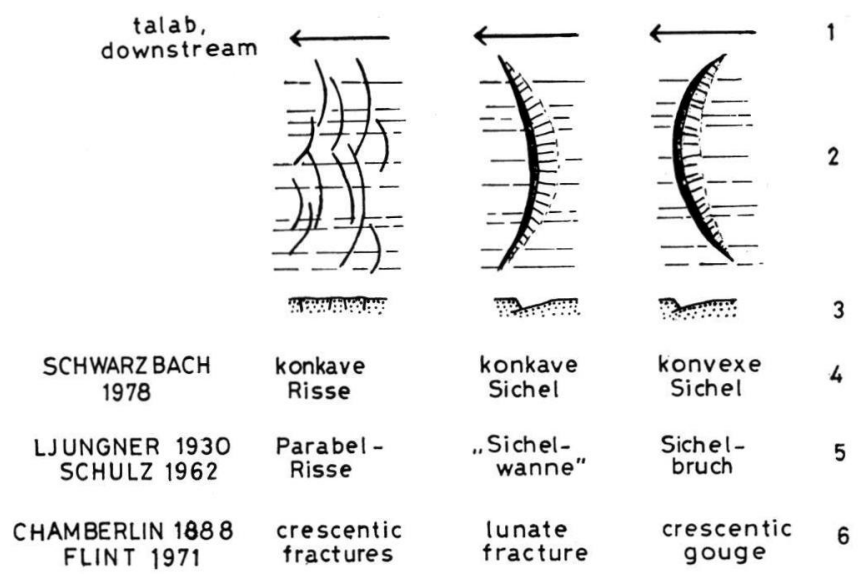

Abb. 4: Ubersicht der Sichelmarken s. 1. - 1 Richtung der Gletscherbewegung, 2 Grundriß der drei Haupttypen (mit Gletscherschrammen), 3 dazugehörige Querschnitte, 4-6 Nomenklatur nach Schwarzbach (diese Arbeit). Ljungner und Schulz ("Sichelwanne" an dieser Stelle nach Schulz, nicht nach LJUNGNeR), Chamberlin (und andere) bis Flint. - Systematic representation of crescentic marks s.l. -1 direction of ice-movement, 2 outline of the 3 main types (with glacial striation), 3 profiles, 4-6 nomenclature after Schwarzbach (this paper), LJUNGNER and Schulz, Chamberlin (and others) to Flint. 
Gilbert). Der andere Bogen ist eine steile, sekundäre Bruchfläche. Beide Brüche begrenzen das herausgebrochene Gesteinsmaterial; der Grundriß und die schräge Eintiefung der „Sichel" kommen auf diese Weise zustande. Nur di es e Gruppe der bogenförmigen Marken kann man also als e igent liche Sichelmarken oder Sichelmarken s. str. bezeichnen. Doch lassen sich alle bogenförmigen Marken einschließlich der „Risse“ als Sichelmarken s. 1. zusammenfassen.

Die bisher übliche Bezeichnungsweise der verschiedenen möglichen Marken ist nicht sehr glücklich. Das gilt für die englische, meist auf Chamberlin zurückgehende Nomenklatur wie für die deutsche, die LJUNGNER geprägt und Schulz übernommen hat (Tab. 1 u. Abb. 4). "Crescentic gouge“, „lunate fracture", „crescentic fracture" geben ja keinen direkten sprachlichen Hinweis für ihre Unterscheidung. Das gleiche gilt für „Sichelbruch“ und "Sichelwanne" (wobei die großen Sichelwannen von LJUNGNER zudem keine glazigenen Schürfmarken zu sein scheinen; sie wären also nicht mit den lunate fractures zu identifizieren). Daher wurde in der Tab. 1 eine neu e Nomenklatur hinzugefügt, die einfacher und übersichtlicher ist. Sie legt das Hauptgewicht auf die Orientierung der Bögen. Dabei sind die nach „vorn“ („talab“) konvexen Marken als konvexe Sichelm a rke n (oder einfach: konvexe Sicheln) bezeichnet (Abb. 5), die entgegengesetzt orientierten Sicheln als k on k a ve S i che $1 \mathrm{~m}$ a rke n (konkave Sicheln, Abb. 6). Die bogenförmigen Risse („Parabelrisse“ bei LJUNGNER) zeigen - soweit aus der Literatur und den eigenen Beobachtungen zu entnehmen ist - stets mit ihrer konkaven Wölbung nach vorn. Sie entsprechen in dieser Beziehung also den konkaven Sicheln, und man kann sie sinngemäß als k o nkave $\mathrm{R}$ is se bezeichnen (Abb. 2 b, 4, 5, 7).

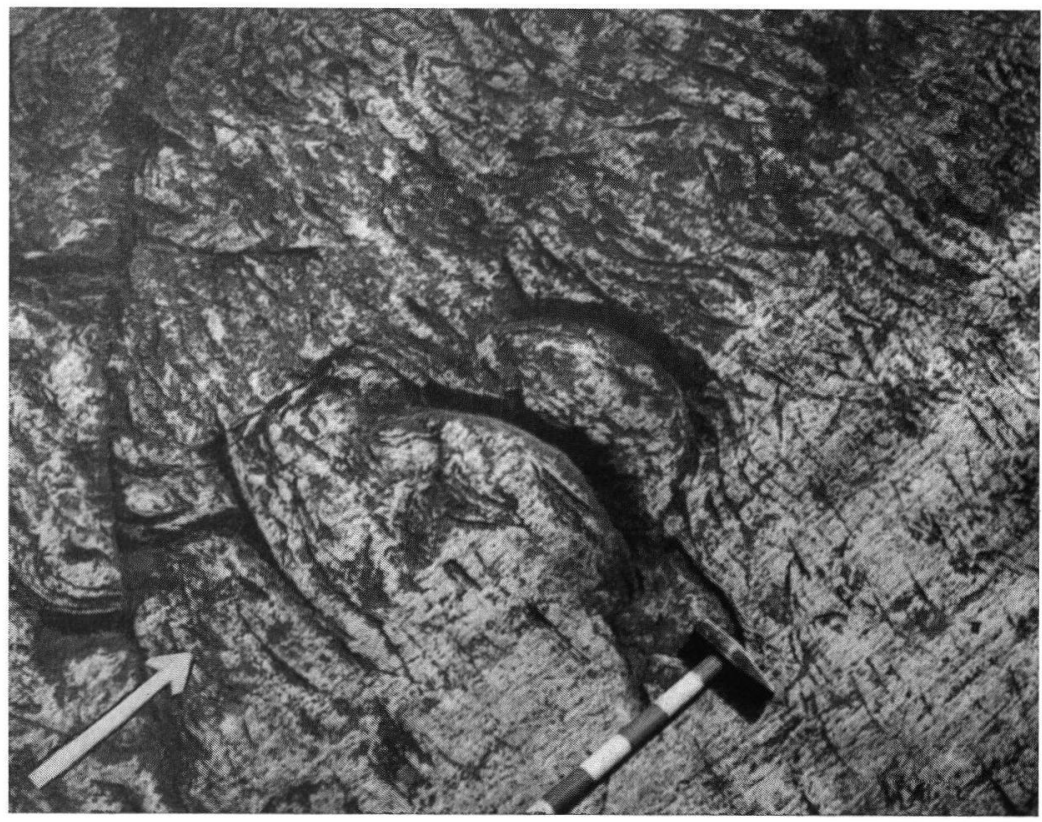

Abb. 5: Zwei konvexe Sicheln; zahlreiche konkave Risse. Neigung (ca. $30^{\circ}$ ) des eisgeschrammten Untergrundes nach rechts unten. Pfeil = Eisbewegung. - Two convex crescents, many concave fractures. Inclination $\left(c, 30^{\circ}\right)$ of the ice-striated bedrock surface to the right-below; arrow = ice movement. 


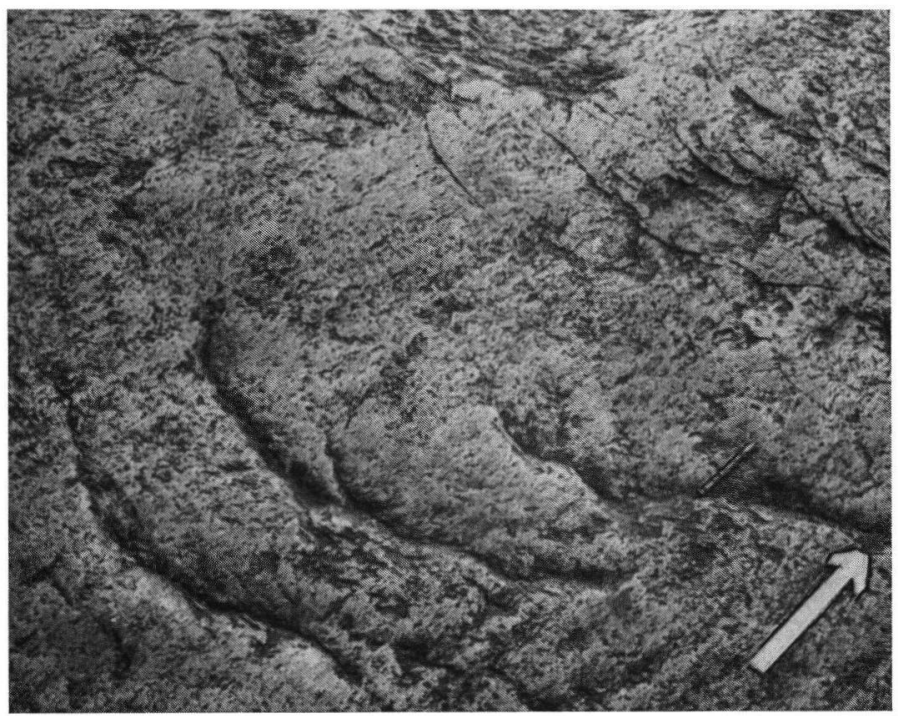

Abb. 6: Einige große, aber nicht besonders regelmäßige konkave Sicheln (Maßstab: Bleistift, nahe dem Pfeil); zahlreiche konkave Risse. Neigung (ca. 30 $0^{\circ}$ ) des eisgeschrammten Untergrundes nach links unten; Pfeil = Eisbewegung. - Some big, but not very regular concave crescents (scale: pencil, near the arrow); many concave fractures. Inclination $\left(c .30^{\circ}\right)$ of the striated bedrock surface to the left-below; arrow = ice-movement.

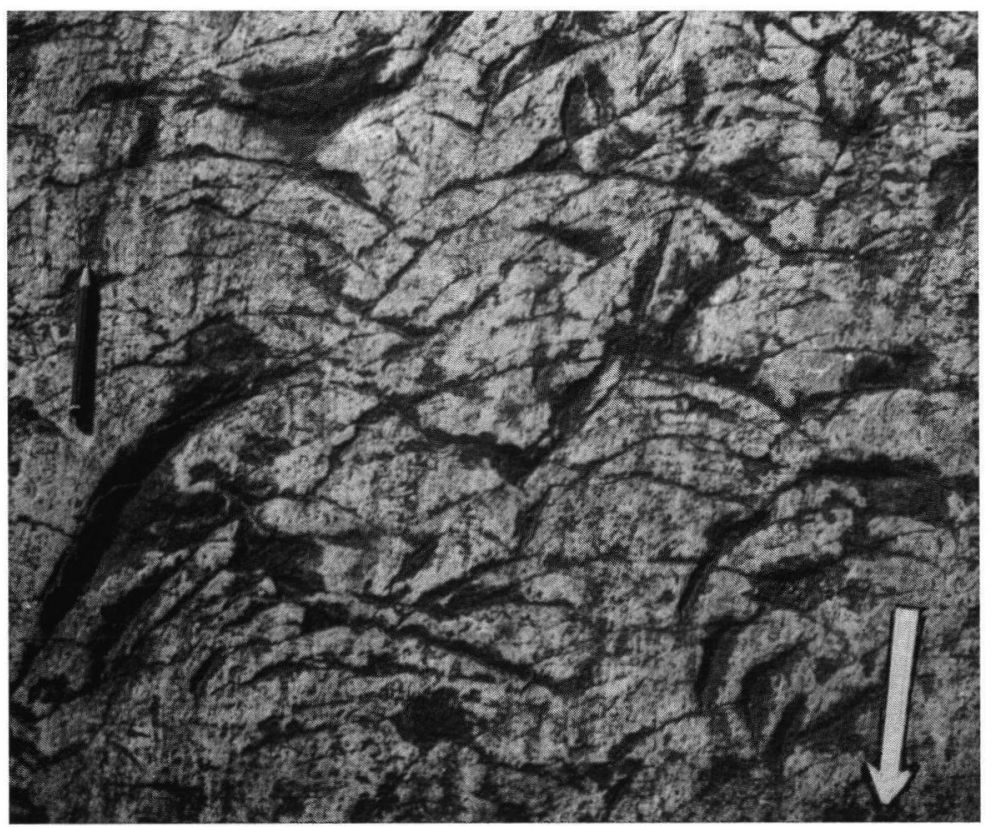

Abb. 7: Zahlreiche konkave Risse; z. T. gehen sie in konkave Sicheln über (Maßstab: Bleistift, links). Neigung (ca. 5-10 $0^{\circ}$ ) des eisgeschrammten Untergrundes nach links. Pfeil = Eisbewegung. - Many concave fractures; partly transitions to crescents (scale: pencil, to the left). Inclination of the ice-striated bedrock surface c. 5-100 to the left; arrow = ice-movement. 
Die konvexen Sichelmarken sind (als crescentic gouges, Sichelbrüche) oft beschrieben worden und ebenso die konkaven Risse (crescentic fractures, Parabelrisse); dagegen als Seltenheiten die konkaven Sicheln (lunate fractures). Die Konkavrisse und Konkavsicheln sind im Extremfall scharf zu trennen. Doch es gibt auch Übergänge von dünnen Linien zu solchen Bögen, bei denen "punktweise" etwas Material herausgebrochen ist (Abb. 7) bis schließlich zu vollkommenen Sicheln. Im allgemeinen tendieren, wie schon HaRris feststellte, die konkaven Risse mehr zu hyperbolischen Formen als die eigentlichen Sichelmarken.

Ein wichtiges Merkmal ist die Neigungsrichtung des primären Hauptbruches (principal fracture). Wie schon erwähnt, ist er bei den konvexen Sicheln flach geneigt, und zwar in Richtung der Eisbewegung. Das gleiche dürfte für die konkaven Sicheln gelten. Man kann auch sagen, daß bei beiden Sichel-Arten der steilere Rand normalerweise „vorn“ liegt.

HARRIS hatte angenommen, daß auch bei den übrigen Marken der primäre Bruch stets „talab“ geneigt ist (wenn auch z. T. recht steil). Das würde ein wichtiges Indiz für die Rekonstruktion der Eisbewegung sein. Dreimanis (1953) zeigte aber, daß mindestens die konkaven Risse dieser Regel nicht folgen. Sie bestehen ja, im Gegensatz zu den Sicheln, nur aus e in e $m$ Bruch, und der steht oft fast vertikal. Die Neigung geht aber dabei nach den Untersuchungen von Dreimanis häufiger talauf als talab, entgegen der Verallgemeinerung von HARRIS.

Die eigentlichen Sichelmarken treten relativ vereinzelt auf, wenn auch manchmal in (nicht sehr regelmäßigen) lockeren Reihen oder Staffeln. Ganz anders die (konkaven) Risse: man findet sie oft scharenweise und einander berührend. LAHEE hat 1912 vom Northey Hill, Lisbon, N. H., überhaupt nur konkave Risse beschrieben. In den Koli-Bergen dürfte gelegentlich das Verhältnis konkaver Marken (in der Hauptsache konkave Risse) zu konvexen Marken zahlenmäßig in der Größenordnung von $100: 1$ sein!

\section{Entstehung der Sichelmarken}

Die Entstehung der bogenförmigen Marken hat schon GiLbert plausibel zu erklären versucht (vgl. zuletzt Schulz 1967). Ein großes Geschiebe, das am oder nahe am Untergrund des Gletschers transportiert wird, erzeugt einen Druckkegel, dessen Achse je nach dem Verhältnis von Eismächtigkeit zu Transportenergie schräg liegt. Er schneidet die Gesteinsoberfläche in einem Kegelschnitt. Vor der Achse herrscht Pressung, dahinter Zerrung. Bei kleinem Einfallswinkel entsteht in der Bewegungsrichtung des Eises ein flachgeneigter primärer Bruch, der zum Ausbrechen einer flachen, sichelförmigen Gesteinsscheibe von dreieckigem Querschnitt führt: konvexe Sichelmarke. Im Bereich der Zerrung bilden sich umgekehrt-orientierte, konkave Risse mit steiler Neigung des Bruches (Abb. 8). Inwieweit sich das überraschend kleine Zahlenverhältnis zwischen Konvexmarken und Konkavrissen, das man im Gelände beobachtet, sowie die Bildung der konkaven Sichelmarken damit befriedigend erklären läßt, wage ich nicht zu entscheiden.

Alle bogenförmigen Marken in den Koli-Bergen entstanden auf massigem Quarzit, also einem harten, spröden Gestein mit weitständiger Klüftung. Quarzit wird auch von anderen Beobachtern als bevorzugtes Material erwähnt.

Doch gibt es auch Quarzite ohne Sichelmarken. Als Beispiel seien die gleichfalls präcambrischen Quarzite bei Ruka angeführt (nördlich von Kuusamo, 66 $16^{\prime} \mathrm{n}$. Br., $29^{\circ} 11^{\prime}$ ö. L.). Sie bilden wie die der Koli-Berge auffällige morphologische Härtlinge und sind manchmal eingeschrammt, aber sie zeigen keinerlei Sichelmarken - ganz offensichtlich wegen der intensiven, engständigen Zerklüftung der Quarzite (im cm-Abstand!). 


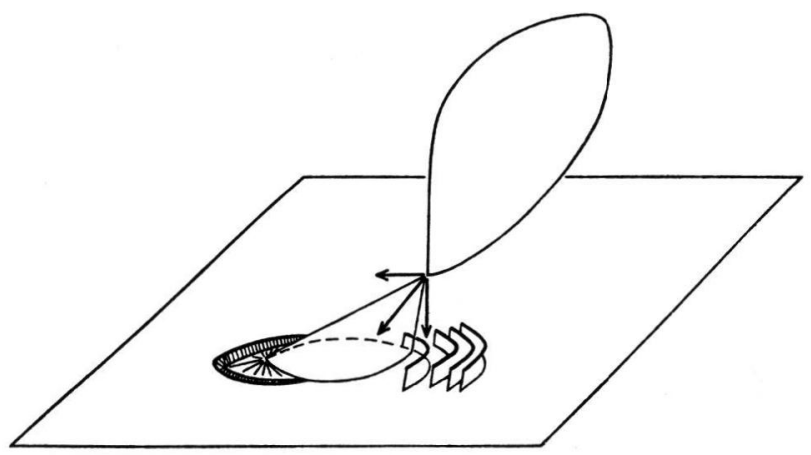

Abb. 8: Schema zur Entstehung von Konvex- und Konkav-Marken. Ein glaziales Geschiebe erzeugt beim Transport einen schräg liegenden Druck-Kegel. Dieser schneidet den Felsuntergrund in einem Kegelschnitt. Es entstehen im Pressungs-Bereich konvexe, im Zerrungs-Bereich konkave Sichelmarken. Aus W. Schulz 1967. - Formation of convex and concave marks (schematically). An erratic boulder produces an inclined pressure-cone which intersects the bedrock-plane in a conic-section; convex marks come into existence in the pressure-region, concave ones in the tensile region. From W. SCHULz 1967.

Ein lehrreiches Einzelbeispiel für den Einfluß des Gesteins sah ich an der Straße Varkaus-Mikkeli, $25 \mathrm{~km}$ nordöstlich von Mikkeli. Ein riesiger Rundhöcker wird dort aus granatführendem, stark schieferigem Gneis aufgebaut mit Adern von hellem, aplitischem Granit. Der Gneis weist nicht gerade hervorragende, aber immerhin merkbare Schrammung auf - (konkave) Sichelmarken finden sich jedoch nur auf einer $1 \mathrm{~m}$ breiten Aplit-Ader!

\section{Die Sichelmarken als Klimazeugen}

Die Frage, ob man mit glazigenen Konkav- und Konvexmarken die Herkunftsrichtung von Gletschereis bestimmten kann, muß man prinzipiell bejahen. Bei den Sichelmarken s. str. kann man zwar nicht aus der Bogenlage allein entscheiden, in welcher Richtung es zur Gletscherzunge ging; denn es läßt sich zunächst nicht sagen, ob es sich um Konvex- oder Konkavsicheln handelt. Wohl aber gibt die flache, talab gerichtete Neigung des primären Bruches einen Hinweis dafür. Für die eigentlichen Sichelmarken ist also das Ergebnis, zu dem HARRIs kam, richtig. Aber es gilt nicht für die bogenförmigen Risse; bei diesen wechselt die Neigung der (steilen) Bruchfläche. Trotzdem können diese Risse unter Umständen besonders wichtig sein; denn sie sind, soweit bekannt, stets Konkavmarken. Ihr haufenweises, eng gedrängtes Auftreten unterscheidet sie von allen andern bogenförmigen Marken.

Bei einer einzeln en Marke kann die Entcheidung, woher das Eis kam, unsicher sein. Aber das gilt für andere Klimazeugen ja ebenfalls: eine einzelne Beobachtung braucht nicht eindeutig zu sein.

Bei der Rekonstruktion jungpleistozäner Talgletscher bedarf es natürlich der Sichelmarken nicht. Das ist wohl auch der Grund, daß Beobachtungen aus dem Alpenbereich ganz zurücktreten, soweit ich sehe. Zum mindesten die neueren „Klassiker“ der alpinen Gletscherforschung, Alb. Heim und R. v. Klebelsberg, erwähnen in den entsprechenden Kapiteln ihrer Handbücher nichts. Bei den großen präkänozoischen Vereisungen können Sichelmarken jedoch von ausschlaggebender Wichtigkeit sein. So haben CROwELL und Frakes $(1970,1971,1972)$ bei den permokarbonischen Vereisungen in Südafrika und Antarktika auf solche Vorkommen und ihre Wichtigkeit für die Rekonstruktion der Gletscher- 
bewegung hingewiesen. Wahrscheinlich gibt es noch andere entsprechende Beispiele; ich habe die weit verstreute Literatur nicht daraufhin durchgesehen. Man darf im übrigen vermuten, daß im Gelände nicht immer auf Sichelmarken geachtet wurde - nur wenige „präquartäre“ Gletscherforscher sind mit den quartären Vereisungsspuren genügend vertraut.

Im übrigen sind die Sichelmarken aber auch bei allgemeinen Untersuchungen über den Mechanismus des Geschiebetransports, also in der allgemeinen Glaziologie, von erheblichem Interesse. -

Die vorliegende kleine Untersuchung wurde „nebenbei“ durchgeführt. Sie berührt nur einzelne Fragen, die sich aus dem Thema ergeben, und ist auch regional ganz unvollständig. Sie gibt aber vielleicht die Anregung zu weiteren Arbeiten.

$\mathrm{D}$ a $\mathrm{n}$ k. Für freundliche Informationen bin ich den finnischen Kollegen Prof. J. DonneR und Prof. P. AlHonen in Helsinki zu Dank verpflichtet.

\section{Schriftenverzeichnis}

Chamberlin, T. C. (1888): The rock-scorings of the Great Ice invasions. - 7th Ann. Rep. U.S. Geol. Surv. 1885-86: 147-248; Washington.

Crowell, J. C. \& Frakes, L. A. (1972): Late Paleozoic glaciation: V, Karroo Basin. - Geol. Soc. Amer., 83, 2887-2912; Boulder.

Dreimanis, A. (1953): Studies of friction cracks along shores of Circus Lake and Kasakokwog Lake, Ontario. - Amer. J. Sci., 251: 769-783; New Haven.

Flint, R. F. (1971): Glacial and Quaternary geology. — 892 S.; New York etc. (J. Wiley).

Frakes, L. A. \& Crowell, J. C. (1970): Late Paleozoic glaciation: II, Africa. - Geol. Soc. Amer. Bull., 81, 2261-2286; Boulder.

- Matthews, J. L. \& Crowell, J. C. (1971): Late Paleozoic glaciation: III, Antarctica. Geol. Soc. Amer. Bull., 82, 1581-1604; Boulder.

Gilbert, G. K. (1905): Crescentic gouges on glaciated surfaces. - Bull. Geol. Soc. Amer., 17: 303-316; New York.

Harris, S. E. (1943): Friction cracks and the direction of glacial movement. - J. Geol. 51: $244-$ 258; Chicago.

LAHEE, F. H. (1912): Crescentic fractures of glacial origin. - Amer. J. Sci. (4) 33: 41-44; New Haven.

LJUnGNer, E. (1930): Spaltentektonik und Morphologie der schwedischen Skagerrak-Küste. III. Bull. Geol. Inst. Univ., Upsala, 21: 255-478; Upsala.

Окко, V. (1950): Friction cracks in Finland. - Bull. Comm. Géol. Finld., 150: 45-50; Helsinki.

Schulz, W. (1962): Sichelförmige Marken auf Quarzitgeschieben von Feldberg in Mecklenburg und Halle (Saale). - Geologie, 11, 1102-1106; Berlin.

- (1967): Über glazigene Schrammen auf dem Untergrund und sichelförmige Marken auf Geschieben in Norddeutschland. - Geogr. Ber., 43: 125-142; Berlin. 